\title{
Low-Complexity Orthogonal Spectral Signal Construction for Generalized OFDMA Uplink With Frequency Synchronization Errors
}

\author{
Zhongren Cao, Member, IEEE, Ufuk Tureli, Member, IEEE, and Yu-Dong Yao, Senior Member, IEEE
}

\begin{abstract}
In orthogonal frequency-division multiplexing, the total spectral resource is partitioned into multiple orthogonal subcarriers. These subcarriers are assigned to different users for simultaneous transmission in orthogonal frequency-division multiple access (OFDMA). In an unsynchronized OFDMA uplink, each user has a different carrier frequency offset (CFO) relative to the common uplink receiver, which results in the loss of orthogonality among subcarriers and thereby multiple access interference. Hence, OFDMA is very sensitive to frequency synchronization errors. In this paper, we construct the received signals in frequency domain that would have been received if all users were frequency synchronized. A generalized OFDMA framework for arbitrary subcarrier assignments is proposed. The interference in the generalized OFDMA uplink due to frequency synchronization errors is characterized in a multiuser signal model. Least squares and minimum mean square error criteria are proposed to construct the orthogonal spectral signals from one OFDMA block contaminated with interference that was caused by the CFOs of multiple users. For OFDMA with a large number of subcarriers, a low-complexity implementation of the proposed algorithms is developed based on a banded matrix approximation. Numerical results illustrate that the proposed algorithms improve the system performance significantly and are computationally affordable using the banded system implementation.
\end{abstract}

Index Terms-Banded matrix, carrier frequency offset (CFO), least squares (LS), minimum mean-square error (MMSE), multiple access, orthogonal frequency-division multiplexing (OFDM), synchronization.

\section{INTRODUCTION}

$\mathbf{F}$ UTURE wireless systems are envisioned to provide reliable and high-speed multimedia services to multiple users simultaneously. Among the wireless technologies proposed so far, orthogonal frequency-division multiplexing (OFDM) has recently garnered intensive research effort. OFDM was

Manuscript received October 25, 2004; revised January 3, 2006 and May 10, 2006. The work of Z. Cao was supported in part by Ericsson. The work of U. Tureli was supported in part by the U.S. Army under Contract W15QKN05-P-0261, in part by AFOSR under Grant FA9550-05-1-0329, in part by NSF under Grant CNS-0520232, WiNSeC under Army Contract W15QKN05-6-0011, and in part by SINTEL under ONR Grant N00014-15-1-063 at Stevens Institute of Technology. The review of this paper was coordinated by Prof. H. Leib.

Z. Cao is with the California Institute for Telecommunications and Information Technology, University of California, San Diego, La Jolla, CA 92093 USA (e-mail: zcao@soe.ucsd.edu).

U. Tureli and Y.-D. Yao are with the Department of Electrical and Computer Engineering, Stevens Institute of Technology, Hoboken, NJ 07030 USA (e-mail: utureli@stevens.edu; yyao@stevens.edu).

Color versions of one or more of the figures in this paper are available online at http://ieeexplore.ieee.org.

Digital Object Identifier 10.1109/TVT.2007.895601 selected as the physical layer technology for digital audio broadcasting [1] and terrestrial digital video broadcasting [2]. In wireless local area networks, OFDM has also been adopted by IEEE 802.11a [3] and HIPERLAN/2 [4]. Multiple access is a challenging issue in designing OFDM systems to fulfill the requirement of future communications. OFDM can be combined with existing multiple access techniques, such as time-division multiple access [5], frequency-division multiple access (OFDM-FDMA) [6], and code-division multiple access (MC-CDMA) [7].

Apart from the aforementioned methods, the intrinsic orthogonal carrier nature of OFDM provides a unique multipleaccess capability, in which multiple subcarriers are assigned to different users for simultaneous transmission. This is termed as orthogonal FDMA (OFDMA) [8]. In OFDMA, signals from multiple users occupying different subcarriers overlap in the frequency domain. The orthogonality among subcarriers prevents intercarrier interference (ICI), which consequently eliminates multiple access interference (MAI) among users. OFDMA has been selected as one of the physical-layer multiple-access technologies in the recent wireless metropolitan area network standard IEEE 802.16 [9], [10]. OFDMA is robust to multipath fading in wireless channels by introducing a cyclic prefix (CP), as is OFDM [11]. Moreover, the wideband frequency resource can be effectively granulated via orthogonal subcarriers for flexible and optimum resource allocation [12]. A deep faded subcarrier for one user is probably a less attenuated one for another user. Thus, we can optimize system performance by assigning better or less attenuated subcarriers to each user [13]. Furthermore, each user can exploit frequency diversity by spreading its subcarriers across the whole bandwidth.

Similar to OFDM, the performance of OFDMA is also very sensitive to frequency synchronization errors. The carrier frequency offset (CFO) between the transmitter and the receiver results in loss of orthogonality among subcarriers and ICI, which further introduces MAI in OFDMA and degrades system performance [14]. The existence of frequency synchronization errors in the OFDMA uplink is mainly due to oscillator instabilities and/or Doppler shifts [15] as well as downlink frequency synchronization errors. This problem is more serious for OFDMA than OFDM since the former typically has a larger number of subcarriers and a much narrower subcarrier spacing, such as less than $1 \mathrm{kHz}$ in some configurations standardized by IEEE 802.16 [9]. The amount of frequency offset becomes a significant portion of the subcarrier spacing. The mitigation of frequency synchronization errors is thus a crucial task in 
designing OFDMA uplink receivers (URs). The effect of CFO in the OFDMA uplink is different from that in the downlink. Frequency synchronization in OFDMA downlink is a single parameter problem. Many frequency synchronization algorithms proposed for OFDM are applicable for the OFDMA downlink [16]. In OFDMA uplink, each user has a different CFO, which results in a multiple-parameter problem. The received signals at UR are contaminated by the interference among subcarriers, and the transmitted information cannot be retrieved error free even in the absence of noise.

The synchronization error correction in OFDMA uplink includes two steps: 1) CFO estimation; 2) correction. CFO estimation can be performed using frame preambles with specific subcarrier assignment schemes. For example, a blind subspacebased multiuser CFO estimation algorithm was proposed in [17] for interleaved OFDMA uplink. However, various other subcarrier assignment schemes may be used in the data transmission part of each frame. CFO correction for OFDMA uplink with a subband-based subcarrier assignment scheme was investigated in [6] and [18]. However, the proposed algorithms in [6] and [18] are not applicable to a variety of other subcarrier assignment schemes, such as block-random assignment [13], interleaved assignment [14], random assignment [19], and clustered assignment [20], [21]. In [22], an iterative interference cancelation scheme is proposed for OFDMA uplink with the assumption that CFO is small compared with subcarrier spacing. Recently, another OFDMA uplink synchronization scheme was proposed in [15], which also does not rely on any specific subcarrier assignment scheme. However, this scheme assumes that only one user is asynchronous with UR while all the other users have achieved synchronization.

Different from above, a new frequency synchronization error correction scheme is proposed in this paper. This scheme can be applied to OFDMA uplink where multiple users are asynchronous with UR simultaneously and $\mathrm{CFO}$ values can be within half of the subcarrier spacing. The investigation in this paper aims to correct the frequency synchronization errors for OFDMA uplink with arbitrary subcarrier assignment. Ideally, the orthogonality among all subcarriers can be physically restored at UR after users adjust their carrier frequencies using feedback from UR. Nevertheless, the feedback process has several drawbacks. First, it will increase the system overhead. Second, due to feedback delays, the estimates may be outdated for the current data transmission. Third, the feedback links are subject to fading and hence may fail to deliver the estimates correctly. Therefore, we propose an alternative method in which UR directly corrects multiuser synchronization errors without feedback. In large cell systems, while initial ranging and feedback are used for timing and frequency synchronization during system entry registration, periodic feedback is still necessary during normal operation to track the frequency changes [9]. The proposed nonfeedback scheme can reduce the frequency of periodic feedback and improve system efficiency. For small cell systems, a nonfeedback scheme is more attractive since the time offset can be accommodated by CP. Without feedback however, each user cannot adjust its frequency on its own. Hence, it is difficult to physically restore the subcarrier orthogonality at UR. Instead, we turn to constructing orthogonal spectral signals- the frequency-domain signals that would have been received if all subcarriers are orthogonal with respect to each other.

In this paper, a generalization of the various OFDMA subcarrier assignment schemes is presented and referred as generalized OFDMA. Frequency synchronization is studied in the context of generalized OFDMA uplink. We first establish a closed-form signal model to characterize the generation of multiuser interference due to frequency synchronization errors using an interference matrix. Least squares (LS) and minimum mean-square error (MMSE) criteria are used for orthogonal spectral signal construction based on the estimated CFO of each user at UR, respectively. Furthermore, a computationally efficient algorithm implementation is proposed based on the observation that the major interference from one subcarrier only affects its neighboring subcarriers. Similar observations have been reported in [23] and [24] for suppressing the ICI caused by fast time-varying channels in a single-user OFDM system. However, these works assume knowledge of channels, which requires prior frequency synchronization. In this paper, we formulate a banded matrix approximation to the interference matrix to correct multiuser frequency synchronization errors on OFDMA uplink.

The rest of this paper is organized as follows. The signals on the OFDMA uplink and the effects of multiuser frequency synchronization errors are investigated in Section II. In Section III, the signal model and the interference matrix are presented along with the proposed orthogonal spectral signal construction algorithms based on the LS method and the MMSE algorithm, respectively. A computationally efficient implementation with a banded matrix approximation is studied in Section IV. Simulation results are reported in Section V, and conclusions are drawn in Section VI.

\section{Multiuser Signals on OFDMA UPLinK}

In this section, OFDMA systems and multiuser subcarrier assignment are introduced first. An investigation into the effect of multiuser CFO is then carried out, and the objective of the proposed algorithms is formulated.

\section{A. Multiple Access in OFDMA}

The OFDMA system under consideration consists of several users and one UR. The users can be fixed or mobile. The focus of this paper is the uplink transmission, namely from users to UR. Each user is assigned a subset of available subcarriers such that no subcarrier is occupied by more than one user. During transmission, each user modulates only the assigned subcarriers.

Let $N$ denote the total number of subcarriers in the OFDMA system. Among the $N$ subcarriers, $2 M$ subcarriers are available for use $(2 M<N)$. The remaining unused subcarriers are the one at the carrier frequency $f_{c}$ and those on both edges of the spectrum mandated by standards to suppress out-of-band energy [3], [9]. Traditionally, $N$ subcarriers are indexed by the discrete Fourier transform (DFT) order as $\{0,1, \ldots, N-1\}$. In this paper, we focus on the $2 M$ available subcarriers and index them according to their frequency positions during transmission as $\{-M, \ldots,-1,1, \ldots, M\}$. This index scheme is shown in 


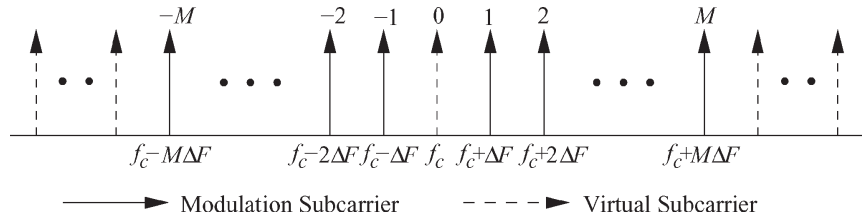

Fig. 1. Modulation subcarrier indices and frequency positions. $f_{c}$ is the carrier frequency, and $\Delta F$ is the subcarrier spacing.

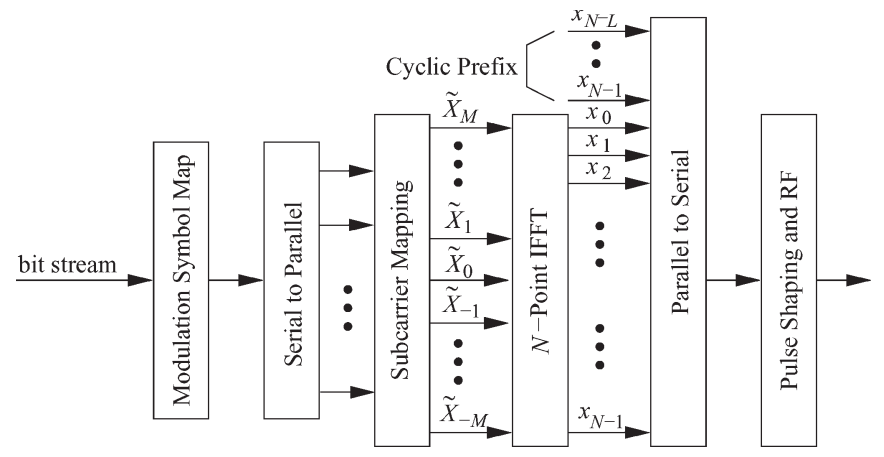

Fig. 2. Single-user OFDMA transmitter block diagram. The superscript $(\cdot)^{(k)}$ is omitted for convenience.

Fig. 1. The unused subcarrier at the carrier frequency corresponds to a dc component in the baseband model and is thus indexed with 0 . Most of the $2 M$ subcarriers are used for data modulation, some of them can be used as pilots for channel estimation, and some of them can be used as null subcarriers as proposed in [25]-[27]. Without loss of generality, the $2 M$ available subcarriers are not differentiated in this paper, since the usage of a subcarrier can be inferred from its modulation symbol.

In order to support multiple access communication, $2 M$ available subcarriers are divided into $Q$ disjoint subchannels, which are denoted as $\mathcal{M}_{q}$, for $q=0,1, \ldots, Q-1$. The partition satisfies the following conditions:

$$
\begin{gathered}
\mathcal{M}=\bigcup_{q=0}^{Q-1} \mathcal{M}_{q}=\{-M, \ldots,-1,1, \ldots, M\} \\
\mathcal{M}_{q} \bigcap \mathcal{M}_{q^{\prime}}=\emptyset, \quad \text { if } q \neq q^{\prime} .
\end{gathered}
$$

Note that (1) and (2) do not assume any specific subcarrier assignment scheme. The combination of both equations is a generalization of various subcarrier assignment schemes in OFDMA. Hence, it is defined as generalized OFDMA.

Let $\mathcal{M}^{(k)}$ denote the subchannel occupied by user $k$. A single user OFDMA transmitter diagram is shown in Fig. 2. A user maps information bits and control bits into modulation symbols. For each OFDMA block, the modulation symbols to be sent are passed through a subcarrier mapping block, which produces $2 M+1$ modulation symbols $X_{m}^{(k)}$ for $m=$ $-M, \ldots, M$. The superscript $(\cdot)^{(k)}$ denotes the assignment to the $k$ th user. Define vector $\psi^{(k)}=\left[\psi_{-M}^{(k)}, \ldots, \psi_{-1}^{(k)}, \psi_{1}^{(k)}, \ldots\right.$, $\left.\psi_{M}^{(k)}\right]^{\mathrm{T}}$ as the indicator vector for the subcarrier allocation of user $k$, and

$$
\psi_{m}^{(k)}= \begin{cases}1, & m \in \mathcal{M}^{(k)} \\ 0, & m \notin \mathcal{M}^{(k)}\end{cases}
$$

Hence

$$
X_{m}^{(k)}=\psi_{m}^{(k)} \cdot X_{m}^{(k)} \quad \text { for } \quad m \in \mathcal{M}
$$

In other words, $X_{m}^{(k)}=0$ for subcarriers that are not assigned to user $k$. Any subcarrier that is used as a null subcarrier is also conceptually assigned to one user and, correspondingly for that $k, X_{m}^{(k)}=0$ and $\psi^{(k)}=1$. The zeroth subcarrier is a virtual subcarrier, hence $X_{0}^{(k)}=0$ for all $k$. Finally, an $N$-point DFT is carried out over the $2 M+1$ modulation symbols that result in $N$ time-domain signal samples $\left\{x_{n}^{(k)}\right\}_{n=0}^{N-1}$ as

$$
x_{n}^{(k)}=\frac{1}{\sqrt{N}} \sum_{m=-M}^{M} X_{m}^{(k)} e^{j 2 \pi m n / N} .
$$

Let $L$ denote the length of $\mathrm{CP}$, which is generated by copying the last $L$ samples of $\left\{x_{n}^{(k)}\right\}_{n=0}^{N-1}$ and inserting in front of $x_{0}^{(k)}$. The resulting $N+L$ signal samples that form one OFDMA block are serially ordered as

$$
\left\{x_{N-L}^{(k)}, \ldots, x_{N-1}^{(k)}, x_{0}^{(k)}, \ldots, x_{N-1}^{(k)}\right\}
$$

and transmitted to UR.

Denote the tap-delayed multipath fading channel between the $k$ th user and UR as $\mathbf{h}^{(k)}=\left[h_{0}^{(k)}, h_{1}^{(k)}, \ldots, h_{L-1}^{(k)}\right]$. The orthogonality of subcarriers enables the OFDMA system to partition the whole frequency band into many narrow bands, each corresponding to a subcarrier and is only subject to a flat fading. Let $\bar{H}_{m}^{(k)}$ denote the channel frequency response between user $k$ and UR on the $m$ th subcarrier of $\mathbf{h}^{(k)}$. Hence

$$
\bar{H}_{m}^{(k)}=\sum_{l=0}^{L-1} h_{l}^{(k)} e^{j 2 \pi m l / N} .
$$

In an ideal noise-free reception without synchronization error, and after the removal of $\mathrm{CP}$, the received signal samples of one OFDMA block at UR are the sum of signals from all users as given by

$$
\Upsilon(n)=\frac{1}{\sqrt{N}} \sum_{k=1}^{K} \sum_{m=-M}^{M} X_{m}^{(k)} \bar{H}_{m}^{(k)} e^{j 2 \pi m n / N}
$$

for $n=0,1, \ldots, N-1$.

\section{B. Synchronization Errors on OFDMA Uplink}

In a practical environment, UR faces synchronization errors in both frequency and time domains. Let $\Delta f^{(k)}$ denote the CFO between user $k$ and UR. The detrimental effect of $\Delta f^{(k)}$ depends on its ratio to the subcarrier spacing $\Delta F$. The normalized $\mathrm{CFO}$ (NCFO) is used to quantify the relationship between $\Delta f^{(k)}$ and $\Delta F$ and is defined as

$$
\xi^{(k)}=\frac{\Delta f^{(k)}}{\Delta F} .
$$

The range of NCFO depends on system design parameters, such as carrier frequency, oscillator stability, mobile speeds, signal 
bandwidth, and fast Fourier transform (FFT)/inverse FFT size. Without loss of generality, NCFO can be divided into an integer part and a fractional part, i.e.,

$$
\xi^{(k)}=A+\varepsilon
$$

where $A$ is an integer, and $\varepsilon \in[-0.5,0.5)$. The integer part only causes a shift of subcarrier indices, while the fractional part results in loss of orthogonality and ICI [28]. The integer part of NCFO can be compensated by each user during downlink initial acquisition [29]. Hence, $\left|\xi^{(k)}\right|<0.5$ is assumed in this paper for the range of NCFO in OFDMA uplink, i.e., $\Delta f^{(k)}$ is limited to half of the subcarrier spacing.

Signals from multiple users also arrive at UR at different time instances, which introduce time offsets between users and the UR receiving window. These time offsets result in the misalignment of signals for one OFDMA block from multiple users and may cause interblock interference [17]. The impairment of synchronization errors changes the signal $\{\Upsilon(n)\}_{n=0}^{N-1}$ in (6) to

$$
\begin{aligned}
\Upsilon(n)= & \frac{1}{\sqrt{N}} \sum_{k=1}^{K} e^{j 2 \pi \theta^{(k)}} e^{j 2 \pi \xi^{(k)} n / N} \\
& \cdot \sum_{m=-M}^{M} X_{m}^{(k)} \bar{H}_{m}^{(k)} e^{j 2 \pi m\left(n-n_{d}^{(k)}\right) / N}
\end{aligned}
$$

where $\theta^{(k)}$ is the initial phase from previous OFDMA blocks due to synchronization errors, and $n_{d}^{(k)}$ is the time offset between user $k$ and UR.

The precision requirement of time synchronization can be relaxed by designing the length of $\mathrm{CP}$ to accommodate both maximum channel delay spread and maximum time offset among all users [30]. By this design, the time offsets between users and UR only introduce circular shifts of signals within the OFDMA block. Therefore, the OFDMA system is quasisynchronized in time domain, and the offset $n_{d}^{(k)}$ and the initial phase $\theta^{(k)}$ can be incorporated into the channel frequency response as

$$
H_{m}^{(k)}=\bar{H}_{m}^{(k)} e^{j 2 \pi\left(\theta^{(k)}-m n_{d}^{(k)} / N\right)}
$$

and (9) is thus simplified to

$$
\Upsilon(n)=\frac{1}{\sqrt{N}} \sum_{k=1}^{K} e^{j 2 \pi \xi^{(k)} n / N} \sum_{m=-M}^{M} X_{m}^{(k)} H_{m}^{(k)} e^{j 2 \pi m n / N} .
$$

After DFT demodulation, the signal on the $m$ th subcarrier at UR is

$$
\begin{aligned}
& G_{m}= \frac{1}{\sqrt{N}} \sum_{n=0}^{N-1} \Upsilon(n) e^{-j 2 \pi m n / N} \\
&=\frac{1}{N} \sum_{m^{\prime}=-M}^{M}\left\{\sum_{k=1}^{K} X_{m^{\prime}}^{(k)} H_{m^{\prime}}^{(k)}\right. \\
&\left.\cdot\left(\sum_{n=0}^{N-1} e^{\left.j 2 \pi\left[\left(m^{\prime}-m\right)+\xi^{(k)}\right] n / N\right)}\right)\right\} .
\end{aligned}
$$

Let $S_{m}=\sum_{k=1}^{K} X_{m}^{(k)} H_{m}^{(k)}$. Note that $X_{0}^{(k)}=0$ for all users, hence $S_{0}=0$. Based on (3), $S_{m}=X_{m}^{\left(k_{0}\right)} H_{m}^{\left(k_{0}\right)}$ for $m \in$ $\mathcal{M}^{\left(k_{0}\right)}$. Clearly, $S_{m}$ is only determined by the transmitted signal and the channel of a single user $k_{0}$, who occupies the $m$ th subcarrier. If all users are frequency synchronized with UR, i.e., $\xi^{(k)}=0$ for all $k \in[1, K]$, it can be shown from (11) that

$$
G_{m}=X_{m}^{\left(k_{0}\right)} H_{m}^{\left(k_{0}\right)}=S_{m} \quad \text { for } m \in \mathcal{M}^{\left(k_{0}\right)} .
$$

Therefore, $S_{m}$ is the ideal spectral signal received on the $m$ th subcarrier when all subcarriers are orthogonal to each other. Nevertheless, if there are CFOs between different users and UR, $\xi^{(k)} \neq 0$ for some or all $k$. Equation (11) can be expressed as

$$
G_{m}=\sum_{m^{\prime}=-M}^{M} \mathbf{i}_{m, m^{\prime}} \mathbf{u}_{m^{\prime}}^{\mathrm{T}}
$$

where $\mathbf{u}_{m^{\prime}}=\left[X_{m^{\prime}}^{(1)} H_{m^{\prime}}^{(1)}, X_{m^{\prime}}^{(2)} H_{m^{\prime}}^{(2)}, \ldots, X_{m^{\prime}}^{(K)} H_{m^{\prime}}^{(K)}\right], \mathbf{i}_{m, m^{\prime}}=$ $\left[I_{m, m^{\prime}}^{(1)}, I_{m, m^{\prime}}^{(2)}, \ldots, I_{m, m^{\prime}}^{(K)}\right]$, and $(\cdot)^{\mathrm{T}}$ denotes the vector or matrix transpose. From (11), it can be shown that

$$
I_{m, m^{\prime}}^{k}=\eta \cdot \frac{\sin \left(\pi\left[\left(m^{\prime}-m\right)+\xi^{(k)}\right]\right)}{N \sin \left(\pi\left[\left(m^{\prime}-m\right)+\xi^{(k)}\right] / N\right)}
$$

where $k$ is determined by $m^{\prime} \in \mathcal{M}^{(k)}$, and $\eta$ is given by

$$
\eta=\exp \left(j \pi\left[\left(m^{\prime}-m\right)+\xi^{(k)}\right](N-1) / N\right) .
$$

Equation (14) is the spectrum leakage from the $m^{\prime}$ th subcarrier on the $m$ th subcarrier due to the $k$ th user's CFO. In fact, (13) is a generalized uplink signal model for multicarrier systems with frequency synchronization errors. If $K=1$, (13) reduces to the signal model for a single-user OFDM system with CFO that is presented in [31] and [32]. For MC-CDMA systems, all the entries in $\mathbf{u}_{m^{\prime}}$ are nonzero. For OFDMA systems, if $m^{\prime} \in$ $\mathcal{M}^{(k)}$, only the $k$ th element of $\mathbf{u}_{m^{\prime}}$ is nonzero, and

$$
\begin{aligned}
G_{m} & =\sum_{k=1}^{K} \sum_{m^{\prime}=-M}^{M} X_{m^{\prime}}^{(k)} H_{m^{\prime}}^{(k)} I_{m, m^{\prime}}^{(k)} \\
& =\underbrace{S_{m} I_{m, m}^{\left(k_{0}\right)}}_{\tilde{S}_{m}}+\underbrace{\sum_{k=1}^{K} \sum_{\substack{m^{\prime} \in \mathcal{M}(k) \\
m^{\prime} \neq m}} S_{m^{\prime}} I_{m, m^{\prime}}^{(k)}}_{\tilde{I}_{m}}
\end{aligned}
$$

for $m \in \mathcal{M}^{k_{0}} . \tilde{S}_{m}$ is the signal part of $G_{m}$, and $\tilde{I}_{m}$ is the interference part. $\tilde{I}_{m}$ is the summation of the interference from all other subcarriers on the $m$ th subcarrier. In particular, $I_{m, m^{\prime}}^{(k)}$ can be regarded as the normalized interference on the $m$ th subcarrier from the $m^{\prime}$ th subcarrier due to CFO $\xi^{(k)}$, since the actual interference is determined by $S_{m^{\prime}}^{(k)} I_{m, m^{\prime}}^{(k)}$. By iteratively 
applying the triangle inequality, the signal part on the $m$ th subcarrier $\tilde{S}_{m}$ is upper bounded by

$$
\begin{aligned}
\left|\tilde{S}_{m}\right| & =\left|S_{m}\right| \cdot\left|\frac{1}{N} \sum_{n=0}^{N-1} e^{j 2 \pi n \xi^{\left(k_{0}\right)} / N}\right| \\
& <\frac{1}{N}\left|S_{m}\right| \sum_{n=0}^{N-1}\left|e^{j 2 \pi n \xi^{\left(k_{0}\right)} / N}\right|=\left|S_{m}\right| .
\end{aligned}
$$

Therefore, $G_{m}$ is subject to not only interference from all other subcarriers but also degradation of the desired signal on the $m$ th subcarrier due to CFO.

Since different users have unequal CFOs, and the received spectral signals $\left\{G_{m}\right\}_{m \in \mathcal{M}}$ are subject to serious interference, it is difficult to physically recover the orthogonality of all subcarriers. Therefore, the strategy in this paper is to construct the orthogonal spectral signals $\left\{S_{m}\right\}_{m \in \mathcal{M}}$ based on $\left\{G_{m}\right\}_{m \in \mathcal{M}}$ and the estimated CFO $\left\{\xi^{(k)}\right\}_{k=1}^{K}$ at UR. This is equivalent to physically restoring the synchronization and suppressing the interference caused by CFO. Channel estimation can then be applied to find $H_{m}^{(k)}$ for $m \in \mathcal{M}_{q^{(k)}}$ and subsequently $X_{m}^{(k)}$.

\section{Orthogonal Spectral Signal Construction}

In this section, a closed-form matrix-based signal model is proposed for a multiuser OFDMA system. Then, algorithms for orthogonal spectral signal construction are derived.

\section{A. Signal Model and Interference Matrix}

Letting the received signal sequence of a single-user OFDMA block be $\mathbf{r}^{(k)}=\left[\Upsilon^{(k)}(0), \Upsilon^{(k)}(1), \ldots, \Upsilon^{(k)}(N-\right.$ 1) $]^{\mathrm{T}}$, we have

$$
\mathbf{r}^{(k)}=\mathbf{E}^{(k)} \mathbf{W} \mathbf{s}^{(k)}
$$

where $\mathbf{E}^{(k)}$ is an $N \times N$ diagonal matrix given by

$$
\mathbf{E}^{(k)}=\operatorname{Diag}\left\{1, e^{j 2 \pi \xi^{(k)} / N}, \ldots, e^{j 2 \pi \xi^{(k)}(N-1) / N}\right\}
$$

and $\mathbf{s}^{(k)}$ is a $2 M \times 1$ column vector given by

$$
\left[H_{-M}^{(k)} X_{-M}^{(k)}, \ldots, H_{-1}^{(k)} X_{-1}^{(k)}, H_{1}^{(k)} X_{1}^{(k)} \ldots, H_{M}^{(k)} X_{M}^{(k)}\right]^{\mathrm{T}} .
$$

W is an $N \times 2 M$ inverse DFT matrix whose $(n, m)$ th element is $e^{-j 2 \pi n m / N}$ for $n=0,1, \ldots, N-1$ and $m=$ $-M, \ldots,-1,1, \ldots, M$.

Let $\mathbf{s}=\left[S_{-M}, \ldots, S_{-1}, S_{1}, \ldots, S_{M}\right]^{\mathrm{T}}$. According to (12), $\mathbf{s}$ is the ideal orthogonal spectrum signals that we would like to construct in the following, and $\mathbf{s}^{(k)}$ is the contribution to $\mathbf{s}$ from the $k$ th user. Based on (3), we have

$$
\mathbf{s}^{k}=\Psi^{k} \mathbf{s}
$$

where $\boldsymbol{\Psi}^{(k)}=\operatorname{diag}\left[\psi^{(k)}\right]$. Equation (10) can thus be expressed in matrix form as

$$
\mathbf{r}=\sum_{k=1}^{K} \mathbf{r}^{(k)}=\sum_{k=1}^{K} \mathbf{E}^{(k)} \mathbf{W} \mathbf{s}^{(k)}
$$

where $\mathbf{r}=[\Upsilon(0), \Upsilon(1), \ldots, \Upsilon(N-1)]^{\mathrm{T}}$. After applying the DFT operator $\mathbf{W}^{\mathcal{H}}$, where $(\cdot)^{\mathcal{H}}$ denotes the Hermitian matrix, we have

$$
\mathbf{g}=\mathbf{W}^{\mathcal{H}} \mathbf{r}=\sum_{k=1}^{K} \mathbf{W}^{\mathcal{H}} \mathbf{E}^{(k)} \mathbf{W} \mathbf{s}^{(k)}
$$

where $\mathbf{g}=\left[G_{-M}, \ldots, G_{-1}, G_{1}, \ldots, G_{M}\right]^{\mathrm{T}}$. Define $\boldsymbol{\Pi}^{(k)}=$ $\mathbf{W}^{\mathcal{H}} \mathbf{E}^{(k)} \mathbf{W}$. Combining (18) and (20), we have

$$
\mathbf{g}=\sum_{k=1}^{K} \boldsymbol{\Pi}^{(k)} \Psi^{(k)} \mathbf{s}=\mathbf{\Pi} \mathbf{s}
$$

where

$$
\boldsymbol{\Pi}=\sum_{k=1}^{K} \boldsymbol{\Pi}^{(k)} \Psi^{(k)} .
$$

$\Pi$ is a $2 M \times 2 M$ matrix. In order to be consistent with the subcarrier index, the columns of $\Pi$ are also indexed from left to right as $\{-M, \ldots,-1,1, \ldots, M\}$, and the rows are indexed similarly from top to bottom. It can be shown from (15) that the $\left(m, m^{\prime}\right)$ th entry of $\Pi$ is $I_{m, m^{\prime}}^{(k)}$ for $m^{\prime} \in \mathcal{M}^{(k)}$. The $m^{\prime}$ th column of $\Pi$ indicates the interference on all other subcarriers that generated by the $m^{\prime}$ th subcarrier as well as the signal degradation of itself. The $m$ th row of $\Pi$ decomposes the received signal on the $m$ th subcarrier into the interference from all other subcarriers and the degraded signal of itself. Therefore, $\Pi$ can be interpreted as an interference matrix, which operates on the ideal orthogonal spectral signal $\mathbf{s}$ to produce the received signals $\mathbf{g}$ due to the existence of $\left\{\xi^{(k)}\right\}_{k=1}^{K}$. Therefore, (21) is a frequency-domain interference-matrix-based signal model for the generalized OFDMA uplink.

\section{B. Orthogonal Spectral Signal Construction}

The vector of spectral signal samples of an OFDMA block obtained in a noisy environment is

$$
\mathbf{y}=\mathbf{g}+\mathbf{z}=\Pi \mathbf{s}+\mathbf{z}
$$

where $\mathbf{z}=\left[Z_{-M}, \ldots, Z_{-1}, Z_{1}, \ldots, Z_{M}\right]^{\mathrm{T}}$, and $Z_{m}$ is the noise on the $m$ th subcarrier. To construct the orthogonal spectral signals is to construct $s$ from the received $\mathbf{y}$. In this paper, two different criteria are applied: LS and minimum mean squared error. The CFOs $\left\{\xi^{(k)}\right\}_{k=1}^{K}$ are assumed to have been estimated and known to UR; hence, $\Pi$ is available in the following derivation.

1) $L S$ : The linear model of (21) leads to the classical LS criterion, which is to minimize the sum of noise energy in one OFDMA block. The cost function formulated based on the LS criterion is

$$
\mathbf{s}_{\mathrm{LS}}^{\star}=\arg \min _{\mathbf{s}}(\mathbf{y}-\mathbf{\Pi} \mathbf{s})^{\mathcal{H}}(\mathbf{y}-\mathbf{\Pi} \mathbf{s}) .
$$

The solution to (24) is given by

$$
\mathbf{s}_{\mathrm{LS}}^{\star}=\left(\boldsymbol{\Pi}^{\mathcal{H}} \boldsymbol{\Pi}\right)^{-1} \boldsymbol{\Pi}^{\mathcal{H}} \mathbf{y} .
$$


In the Appendix, we show that $\Pi$ is a full rank matrix. Thus, we have

$$
\mathrm{s}_{\mathrm{LS}}^{\star}=\boldsymbol{\Pi}^{-1} \mathbf{y}
$$

2) MMSE: In MMSE, the second-order statistics of the signals and noise are assumed known to UR. Let the noise on each subcarrier be additive white Gaussian with zero mean and variance $\sigma_{n}^{2}$ such that $\mathbb{E}\left[\mathbf{z z}^{\mathcal{H}}\right]=\sigma_{n}^{2} \mathbf{I}$, where $\mathbf{I}$ is a $2 M \times 2 M$ identity matrix, and $\mathbb{E}[\cdot]$ denotes the expectation operator. Let $\mathbf{s}_{\mathrm{MMSE}}^{\star}$ be the MMSE solution of $\mathbf{s}$, and it is given by $\mathbf{s}_{\mathrm{MMSE}}^{\star}=$ $\boldsymbol{\Omega}^{\mathcal{H}} \mathbf{y}$. Based on the orthogonality principle [33], we have

$$
\mathbf{s}_{\mathrm{MMSE}}^{\star}=\mathbf{R} \boldsymbol{\Pi}^{\mathcal{H}}\left(\mathbf{\Pi} \mathbf{R} \boldsymbol{\Pi}^{\mathcal{H}}+\sigma_{n}^{2} \mathbf{I}\right)^{-1} \mathbf{y}
$$

where $\mathbf{R}=\mathbb{E}\left[\mathbf{s s}^{\mathcal{H}}\right]$ is the autocorrelation matrix of the orthogonal spectral signal $\mathbf{s}$. If the average powers of the orthogonal spectral signal on all subcarriers are the same, and hence denoted as $\sigma_{s}^{2}$, we have $\mathbf{R}=\sigma_{s}^{2} \mathbf{I}$. Thus, $\mathbf{s}_{\mathrm{MMSE}}^{\star}$ in (27) can be simplified to

$$
\mathbf{s}_{\mathrm{MMSE}}^{\star}=\boldsymbol{\Pi}^{\mathcal{H}}\left(\boldsymbol{\Pi} \boldsymbol{\Pi}^{\mathcal{H}}+\frac{\sigma_{n}^{2}}{\sigma_{s}^{2}} \mathbf{I}\right)^{-1} \mathbf{y} .
$$

\section{BANDED SYSTEM-EFFICIENT IMPLEMENTATION}

The algorithms described in the previous section may involve significant computational complexity, especially for an OFDMA system with a large number of subcarriers, such as $N=2048$ in IEEE 802.16 [9]. A computationally efficient implementation with affordable performance tradeoff is introduced in this section.

\section{A. Interference Localization and Banded System}

The interference on the $m$ th subcarrier caused by the signal on the $m^{\prime}$ th subcarrier is $S_{m^{\prime}} I_{m, m^{\prime}} . I_{m, m^{\prime}}$ is given in (14) and depends on the relative subcarrier distance $m^{\prime}-m$, the NCFO $\xi^{(k)}$, and the IDFT/DFT operation size $N$. The normalized interference power from the $m^{\prime}$ th subcarrier to the $m$ th subcarrier is

$$
\begin{aligned}
\left|I_{m, m^{\prime}}\right|^{2} & =\frac{\sin ^{2}\left(\pi\left[\left(m^{\prime}-m\right)+\xi^{(k)}\right]\right)}{N^{2} \sin ^{2}\left(\pi\left[\left(m^{\prime}-m\right)+\xi^{(k)}\right] / N\right)} \\
& =\frac{1-\cos \left(2 \pi \xi^{(k)}\right)}{N^{2}\left(1-\cos \left[2 \pi\left(m^{\prime}-m\right) / N+2 \pi \xi^{(k)} / N\right]\right)} .
\end{aligned}
$$

In OFDMA, $N$ is typically large, so $2 \pi \xi^{(k)} / N \approx 0$, and

$$
\left|I_{m, m^{\prime}}\right|^{2} \approx \frac{1-\cos \left(2 \pi \xi^{(k)}\right)}{N^{2}\left(1-\cos \left[2 \pi\left(m^{\prime}-m\right) / N\right]\right)}
$$

In Fig. 3, $\left|I_{m, m^{\prime}}\right|^{2}$ versus $m^{\prime}-m$ for different $\xi^{(k)}$ are plotted. As shown, for $\left|m^{\prime}-m\right|>10,\left|I_{m, m^{\prime}}\right|^{2}$ is below $-30 \mathrm{~dB}$, and for $\left|m^{\prime}-m\right|>20,\left|I_{m, m^{\prime}}\right|^{2}$ is below $-38 \mathrm{~dB}$. The conclusion drawn from Fig. 3 is that the interference caused by the $m^{\prime}$ th

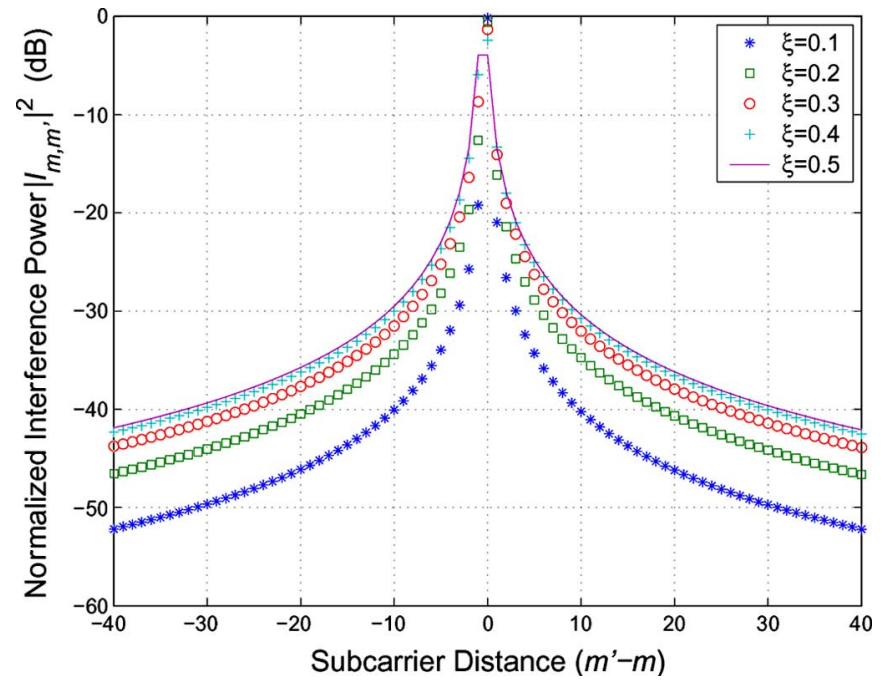

Fig. 3. Normalized interference power of a single subcarrier on its nearby subcarriers.

subcarrier only has significant effects on its nearby subcarriers; for all other subcarriers, the impact of the interference from the $m^{\prime}$ th subcarrier is negligible. In a practical system, a threshold $\tau$ can be introduced as a design parameter. For subcarrier group $\left\{m:\left|m^{\prime}-m\right|>\tau\right\}$, the interference caused by the $m^{\prime}$ th subcarrier on the $m$ th subcarrier is neglected, and the normalized interference $I_{m, m^{\prime}}$ is assumed to be zero. Therefore, the interference generated by subcarrier $m^{\prime}$ is localized to its neighboring subcarrier group $\left\{m:\left|m^{\prime}-m\right| \leq \tau\right\}$.

However, due to the periodicity of trigonometric functions, if $\left|m-m^{\prime}\right|$ approaches $N,\left|I_{m, m^{\prime}}\right|$ becomes significant again. In OFDMA, $\max \left|m^{\prime}-m\right|=2 M$. Therefore, if we constrain $\tau$ such that

$$
\tau<N-2 M
$$

we can separate $\Pi$ into two parts as $\Pi=\Pi_{B}+\Pi_{I}$. Using the same indexing method as that in $\Pi$, the $\left\{m, m^{\prime}\right\}$ th entry of $\boldsymbol{\Pi}_{B}$ is determined by

$$
\boldsymbol{\Pi}_{\mathrm{B}}\left(m, m^{\prime}\right)= \begin{cases}I_{m, m^{\prime}}, & \text { if }\left|m^{\prime}-m\right| \leq \tau \\ 0, & \text { if }\left|m^{\prime}-m\right|>\tau .\end{cases}
$$

Note that $I_{m, m^{\prime}}$ is also the $\left\{m, m^{\prime}\right\}$ th entry of $\boldsymbol{\Pi}$. Hence, $\boldsymbol{\Pi}_{B}$ models the interference that each subcarrier generates on its neighboring subcarriers limited to a distance of $\tau$, while $\boldsymbol{\Pi}_{I}$ records the interference each subcarrier causes on subcarriers that are beyond the distance of $\tau$.

$\Pi_{B}$ only has nonzero entries near its diagonal. This kind of matrix is called Banded Matrix, and $\tau$ is the bandwidth of the matrix [34]. The signal model (23) is, thus, modified to a banded system model as

$$
\mathrm{y}=\Pi_{\mathrm{B}} \mathrm{s}+\Pi_{\mathrm{I}} \mathrm{s}+\mathrm{z}=\Pi_{\mathrm{B}} \mathrm{s}+\mathrm{z}_{\mathrm{B}}
$$

where $\mathbf{z}_{B}=\Pi_{I} \mathbf{s}+\mathbf{z}$. The banded system model is computationally efficient since the inverse of a banded matrix can be implemented with much fewer steps compared with a normal matrix inverse. However, $\boldsymbol{\Pi}_{B}$ only models the major interference each subcarrier generates on its neighboring subcarriers. 


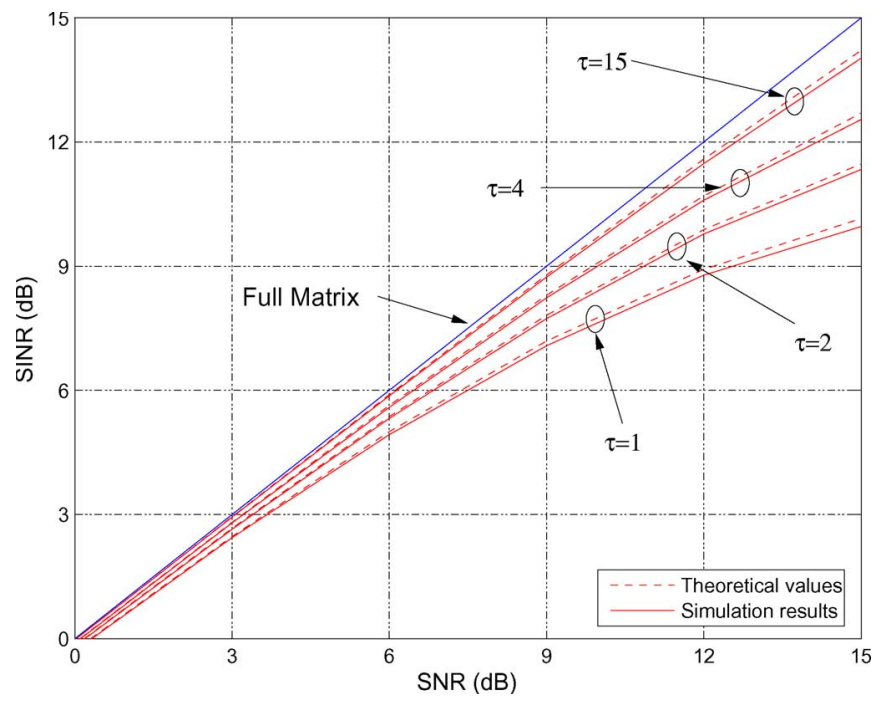

Fig. 4. SINR versus SNR in the banded system model for $N=2048$.

The remaining interference represented by $\Pi_{I} \mathrm{~s}$ on each subcarrier is treated as residual interference. The system noise level is effectively increased. Hence, there is a tradeoff between computational efficiency and system performance by selecting the appropriate $\tau$.

\section{B. Selection of $\tau$}

In a generalized OFDMA uplink, the $m^{\prime}$ th subcarrier can be assigned to any user. Denote the average normalized interference power from the $m^{\prime}$ th subcarrier to the $m$ th subcarrier as $\sigma_{m, m^{\prime}}^{2}=\mathbb{E}\left(\left|I_{m, m^{\prime}}\right|^{2}\right)$. Assuming that $\xi^{(k)}$ for all $K$ users are uniformly distributed over $(-0.5,0.5)$, we have

$$
\begin{aligned}
\sigma_{m, m^{\prime}}^{2} & =\int_{-0.5}^{0.5}\left|I_{m, m^{\prime}}\right|^{2} d \xi^{(k)} \\
& =\frac{1}{N^{2}\left(1-\cos \left[2 \pi\left(m^{\prime}-m\right) / N\right]\right)} .
\end{aligned}
$$

Let $\mathcal{I}_{m, \tau}$ denote the average residual interference on the $m$ th subcarrier in a banded system with matrix bandwidth $\tau$. It can be shown that

$$
\mathcal{I}_{m, \tau}=\sum_{m^{\prime}=u}^{M} \mathcal{E}_{m^{\prime}} \sigma_{m, m^{\prime}}^{2}+\sum_{m^{\prime}=-M}^{v} \mathcal{E}_{m^{\prime}} \sigma_{m, m^{\prime}}^{2}
$$

where $u=\min \{m+\tau, M+1\}$, and $v=\max \{-M-1$, $m-\tau\} . \mathcal{E}_{m^{\prime}}=\mathbb{E}\left[S_{m^{\prime}}^{2}\right]$ is the average received power on the $m^{\prime}$ th subcarrier. If $\mathcal{E}_{m}=\sigma_{s}^{2}$ in all subcarriers, in a banded system with matrix bandwidth $\tau$, the signal-to-interferenceplus-noise ratio (SINR) is

$$
\frac{\mathbf{s}^{\mathcal{H}} \mathbf{s}}{\mathbf{z}_{\mathbf{B}}^{\mathcal{H}} \mathbf{z}_{\mathbf{B}}}=\frac{\sigma_{s}^{2}}{\sum_{m \in \mathcal{M}} \mathcal{I}_{m, \tau} / 2 M+\sigma_{n}^{2}} .
$$

From (33)-(35), we can calculate SINR for systems with different $N$ and $\tau$. The theoretical values are compared with the simulation results and illustrated in Fig. 4 for $N=2048$. It can be seen that (35) closely approaches the simulated SINR. Therefore, based on the required operating signal-to-noise ratio (SNR), the appropriate $\tau$ can be determined by comparing (35) for different $\tau \mathrm{s}$.

\section{Efficient Algorithm Implementation}

In this paper, two main advantages of banded matrix are utilized. First, the low-upper (LU) triangular matrix factorization can be implemented efficiently, and both the resulting upper triangular matrix and the lower triangular matrix are banded matrices with the same matrix bandwidth as the original one [34]. Second, if $\mathbf{A}$ is a banded triangular matrix, solving $\boldsymbol{x}$ for $\mathbf{A} \boldsymbol{x}=\boldsymbol{b}$ can be implemented by forward substitution or backward substitution [34].

The efficient implementation of the LS-based synchronization will be introduced first. From (26), for banded system model, $\mathbf{y}=\Pi_{\mathrm{B}} \mathbf{s}_{\mathrm{LS}}^{\star}$. First, an LU factorization is applied to $\Pi_{B}$, and a lower triangular matrix $\mathbf{L}$ and an upper triangular matrix $\mathbf{U}$ are obtained such that $\boldsymbol{\Pi}_{B}=\mathbf{L} \mathbf{U}$. If the bandwidth of $\boldsymbol{\Pi}_{B}$ is $\tau$, both $\mathbf{L}$ and $\mathbf{U}$ are banded matrices with matrix bandwidth $\tau$. Hence, $\mathbf{y}=\mathbf{L U s}_{\mathrm{LS}}^{\star}$. Let $\hat{\mathbf{s}}=\mathbf{U s}_{\mathrm{LS}}^{\star}$. The second step is to apply banded matrix forward substitution to solve $\hat{\mathbf{s}}$ for $\mathbf{y}=\mathbf{L} \hat{\mathbf{s}}$. In the last step, $\mathbf{s}_{\mathrm{LS}}^{\star}$ is solved by banded matrix back substitution from $\hat{\mathbf{s}}=\mathbf{U s}_{\mathrm{LS}}^{\star}$. The implementations of banded matrix LU factorization, banded matrix forward, and back substitution are presented in [34].

For the MMSE receiver

$$
\mathbf{y}=\left(\boldsymbol{\Pi}_{\mathbf{B}} \boldsymbol{\Pi}_{\mathbf{B}}^{\mathcal{H}}+\frac{\sigma_{n}^{2}}{\sigma_{s}^{2}} \mathbf{I}\right)\left(\boldsymbol{\Pi}_{\mathbf{B}}^{\mathcal{H}}\right)^{-1} \mathbf{s}_{\mathrm{MMSE}}^{\star} .
$$

Since $\Pi_{B}$ is a banded matrix, $\Pi \Pi^{\mathcal{H}}$ is also a banded matrix, and the bandwidth is $2 \tau$. Thus, $\left(\boldsymbol{\Pi}_{B} \boldsymbol{\Pi}_{B}^{\mathcal{H}}+\left(\sigma_{n}^{2} / \sigma_{s}^{2}\right) \mathbf{I}\right)$ is a banded matrix. The algorithm to solve $\mathbf{s}_{\mathrm{MMSE}}^{\star}$ is listed in the following:

1) Let $\widehat{\boldsymbol{\Pi}}_{B}=\boldsymbol{\Pi}_{B} \boldsymbol{\Pi}_{B}^{\mathcal{H}}+\left(\sigma_{n}^{2} / \sigma_{s}^{2}\right) \mathbf{I}$ and LU factorize $\widehat{\boldsymbol{\Pi}}_{B}$ such that $\widehat{\boldsymbol{\Pi}}_{B}=\mathbf{L} \mathbf{U}$.

2) Let $\hat{\mathbf{s}}=\left(\boldsymbol{\Pi}_{B}^{\mathcal{H}}\right)^{-1} \mathbf{s}_{\mathrm{MMSE}}^{\star}$. Solve $\hat{\mathbf{s}}$ by applying banded matrix forward and back substitutions to $\mathbf{y}=\widehat{\boldsymbol{\Pi}}_{B} \hat{\mathbf{s}}$.

3) Solve $\mathbf{s}_{\text {MMSE }}^{\star}$ by $\mathbf{s}_{\text {MMSE }}^{\star}=\boldsymbol{\Pi}_{B}^{\mathcal{H}} \hat{\mathbf{s}}$.

\section{Complexity Analysis}

The proposed algorithms above involve several matrix operations, such as matrix inverse, matrix-matrix multiplication, and matrix-vector multiplication. The computational complexity is analyzed by counting the total number of complex arithmetic operation including addition, subtraction, multiplication, and division that is required to accomplish these matrix manipulations. One complex arithmetic operation is a complex floating point operation (flop), which is the unit for the following analysis.

The dimension of the interference matrix $\Pi$ is $2 M \times 2 M$. For large $M$, the inverse and the matrix-matrix multiplication applied on the full matrix $\Pi$ require $16 M^{3}+\mathcal{O}\left(M^{3}\right)$ flops, while a matrix-vector multiplication will cost $8 M^{2}$ flops. Using a banded system modeling, as shown in Section IV-B, the 
TABLE I

ARITHMETIC OPERATION COUNTS FOR BANDED MATRIX

\begin{tabular}{r|c|c|c|c}
\hline Banded Matrix & Multiplication & Division & Addition & Subtraction \\
\hline \hline Matrix-matrix Production & $2 M(2 \tau+1)^{2}$ & & $2 M(2 \tau+1)^{2}$ & \\
\hline Matrix-vector Production & $2 M(2 \tau+1)$ & & $2 M(2 \tau+1)$ & \\
\hline LU Factorization & $2 M \tau^{2}$ & $2 M \tau$ & & $2 M \tau^{2}$ \\
\hline Forward Substitution & $2 M \tau$ & & & $2 M \tau$ \\
\hline Back Substitution & $2 M \tau$ & $2 M$ & & $2 M \tau$ \\
\hline
\end{tabular}

major matrix operations are LU factorization, forward and back substitutions, two banded matrices multiplication, and banded matrix-vector multiplication. Following [34], the necessary flops for the aforementioned banded matrix operations are calculated and listed in Table $\mathrm{I}$ for $\boldsymbol{\Pi}_{\mathrm{B}}$ with $M \gg \tau$.

1) $L S$ : The LS-based algorithm introduced in Section III is composed of two steps. The first one is the inverse of $\Pi$, and the second step is the matrix-vector multiplication of $\Pi^{-1} \mathbf{y}$. The second step requires $4 M^{2}$ flops complex multiplication and $4 M^{2}$ flops complex addition. Thus, the total complexity is determined by the inverse operation and is $16 M^{3}+\mathcal{O}\left(M^{3}\right)$ flops.

Using banded system modeling, the implementation is carried out in three steps, including LU factorization, forward substitution, and back substitution. $\Pi_{B}$ is a $2 M \times 2 M$ banded matrix with bandwidth $\tau$. The resulting upper and lower triangular matrices $\mathbf{L}$ and $\mathbf{U}$ are both banded with $\tau$. From Table I, the total flops required for the banded LS algorithm is about $2 M\left(2 \tau^{2}+5 \tau+1\right)$.

2) MMSE: For implementation based on the full matrix $\Pi$, the major computational expenses of the MMSE algorithm are matrix-matrix multiplication $\Pi \Pi \Pi^{\mathcal{H}}$, matrix inverse $\left(\Pi \Pi^{\mathcal{H}}+\right.$ $\left.\sigma_{n}^{2} / \sigma_{s}^{2} \mathbf{I}\right)^{-1}$, and another matrix-matrix product of the above inverse matrix with $\Pi^{\mathcal{H}}$. Therefore, the complexity of the proposed MMSE algorithm is on the order of $48 M^{3}+\mathcal{O}\left(M^{3}\right)$ flops.

With the banded system model, the complexity can be reduced using the following steps. The first step is a banded matrix-matrix multiplication $\boldsymbol{\Pi}_{B} \boldsymbol{\Pi}_{B}^{\mathcal{H}}$, which results in a new banded matrix. This operation requires approximately $4 M(2 \tau+1)^{2}$ flops for $M \gg \tau$. The second step is LU factorization and forward and back substitution. Since the banded matrix resulting from the first step has a bandwidth of $2 \tau$, from Table I, it can be shown that the second step requires about $2 M\left(8 \tau^{2}+10 \tau+1\right)$ flops. The last step is a banded matrix and vector production, which will cost $4 M(4 \tau+1)$ flops. Hence, the total complexity is approximately $2 M\left(16 \tau^{2}+26 \tau+5\right)$ flops.

The complexity of both algorithms with different matrix bandwidths $\tau$ for $N=512$ and $M=208$ are compared. The required flops of LS and MMSE for each $\tau$ are illustrated using the bar chart in Fig. 5 in 10-based logarithm.

\section{Simulations And Discussion}

In this section, we will first introduce the simulation setup and metrics used to quantify the performance of synchronization algorithms. Then, the numerical results are reported along with discussions.

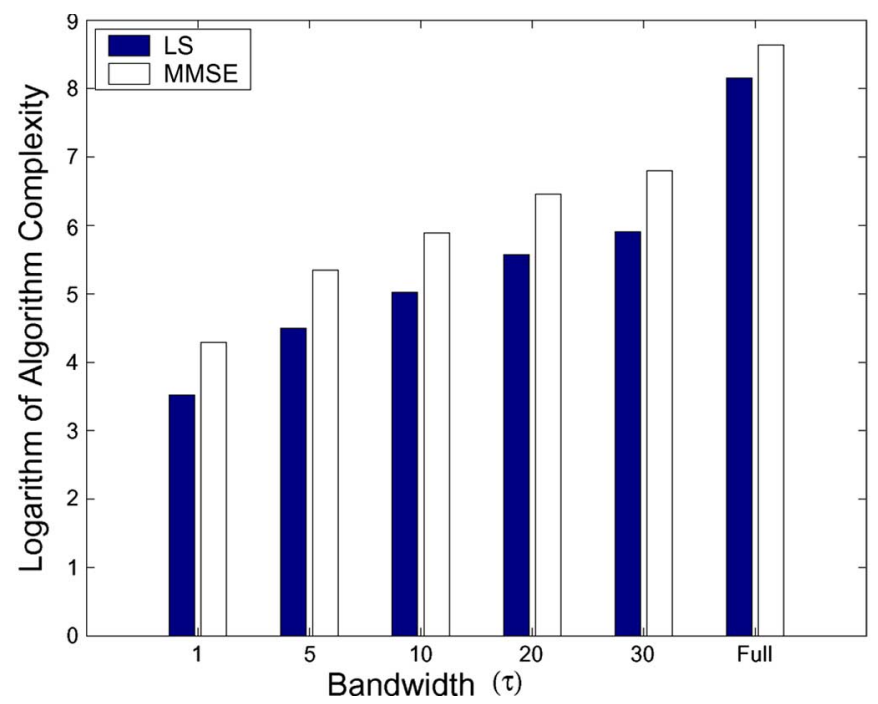

Fig. 5. Algorithm complexity comparison between the full matrix implementation and the banded matrix implementation with different matrix bandwidth $\tau$. The $y$-axis shows the base $10 \operatorname{logarithm}$ of the required total flops corresponding to each case. The flops are valued at $N=512$ and $M=208$ for both LS and MMSE.

\section{A. Simulation Setup}

A 5-MHz OFDMA system with $N=512$ is simulated in a suburban macro cell. The base station is the UR. Multipath channels from each user to the base station antenna are generated according to the Third Generation Partnership Project spatial channel model as described step by step in [35]. In this model, each user's channel consists of $J=6$ multipath components. The average powers for each of the $J$ multipath components are determined by the following exponential power delay profile:

$$
\varrho_{j}^{(k)}=e^{-0.2857\left(d_{j}^{(k)}-d_{0}^{(k)}\right) / \sigma_{\mathrm{DS}}^{(k)}} \cdot 10^{-\beta_{j}^{(k)} / 10}
$$

for $j=0,1, \ldots, 5$, where $\varrho_{j}^{(k)}$ and $d_{j}^{(k)}$ are the power and delay for the $j$ th path of the $k$ th user, $\sigma_{\mathrm{DS}}^{(k)}$ is the root mean square delay spread of the $k$ th user, and $\beta_{j}^{(k)}$ is a shadowing randomization effect on the power of the $j$ th path. The generation of $d_{j}^{(k)}, \sigma_{\mathrm{DS}}^{(k)}$, and $\beta_{j}^{(k)}$ is detailed in [35]. The radius of the macrocell is assumed to be $1500 \mathrm{~m}$. The guard time is selected to be $12.8 \mu$ s to accommodate the multipath delay spread and the time offset among different users due to their different distances to the base station. Each subchannel in the simulated OFDMA system has 52 subcarriers, and each user occupies one subchannel. For $N=512$ and $5 \mathrm{MHz}$, the number of available subcarriers is $2 M=416$, the number of users is $K=8$, and the length of $\mathrm{CP}$ is $L=64$. The performance results under different 
DFT sizes such as $N=256, N=1024$, and $N=2048$ are also evaluated and compared.

In each Monte Carlo realization, all subcarriers are randomly assigned among all users and a set of irregular CFO values. Quaternary phase-shift keying (QPSK) or 16 quadraticamplitude modulation (16QAM) modulation schemes are used to generate a transmitted OFDMA block. Then, a Rayleighfading multipath channel is obtained for each user according to [35]. The average SNR for each user is assumed to be the same by means of power control. All reported numerical results are calculated by averaging over 1000 Monte Carlo realizations. The channels are assumed to be known at the base station. CFO is estimated using a preamble according to [17]. Subcarrier allocation is also known to the base station; thus, $\Pi$ can be constructed after CFO estimation.

To quantify the performance of the proposed algorithm, the following metrics are used as simulation measurements.

1) Normalized Mean-Square Error (NMSE): nmse measures the closeness of the constructed orthogonal spectral signals $s_{L S}^{\star}$ or $s_{\text {MMSE }}^{\star}$ compared with $s$ and is defined as

$$
\text { nmse }=\frac{\mathbb{E}\left[\left|\widehat{S}_{m}-S_{m}\right|^{2}\right]}{\mathbb{E}\left[\left|S_{m}\right|^{2}\right]}
$$

where $\widehat{S}_{m}$ is the estimate of $S_{m}$ after applying the proposed algorithms. nmse measures the average ratio between the power of the signal construction error and the ideal signal power on a subcarrier. The nmse performance shown later is the result of averaging over all subcarriers.

2) Uncoded Bit-Error Rate (BER): Information bits are generated and mapped into the modulation symbols directly in simulation. The uncoded BER is determined to examine the effectiveness of the proposed algorithm independent of any specific error control coding. The modulation symbol is estimated by one tap equalization as $\widehat{X}_{m}^{(k)}=\widehat{S}_{m} / H_{m}^{(k)}$ for $m \in \mathcal{M}^{(k)}$.

\section{B. Numerical Results and Discussion}

Fig. 6 shows the nmse performance of the LS method for an OFDMA system with $N=512$ and $K=8$. The nmse of the MMSE algorithm for the same system is plotted in Fig. 7. The corresponding uncoded BER performance of the LS method is illustrated in Fig. 8, and Fig. 9 is for the MMSE algorithm. For each plot, we show the performance measures for the full matrix implementation as well as the banded system implementation with $\tau=1,5,10,20,30$, respectively. For comparison purposes, the nmse or uncoded BER curve obtained by a direct demodulation without any frequency synchronization effort is also drawn with a dashed line in each figure. Similarly, the nmse and uncoded BER curves of the perfect synchronized case, i.e., the CFO of each user is zero, are also plotted. The nmse in the perfect synchronization case is essentially the noise power.

At low SNR, the nmse of the MMSE algorithm is far better than that of the LS method, while the nmse of the two algorithms are close to each other at high SNR. The uncoded BER performances of the two algorithms are similar for both high and low SNRs. Even with $\tau=1$, the banded system implemen-

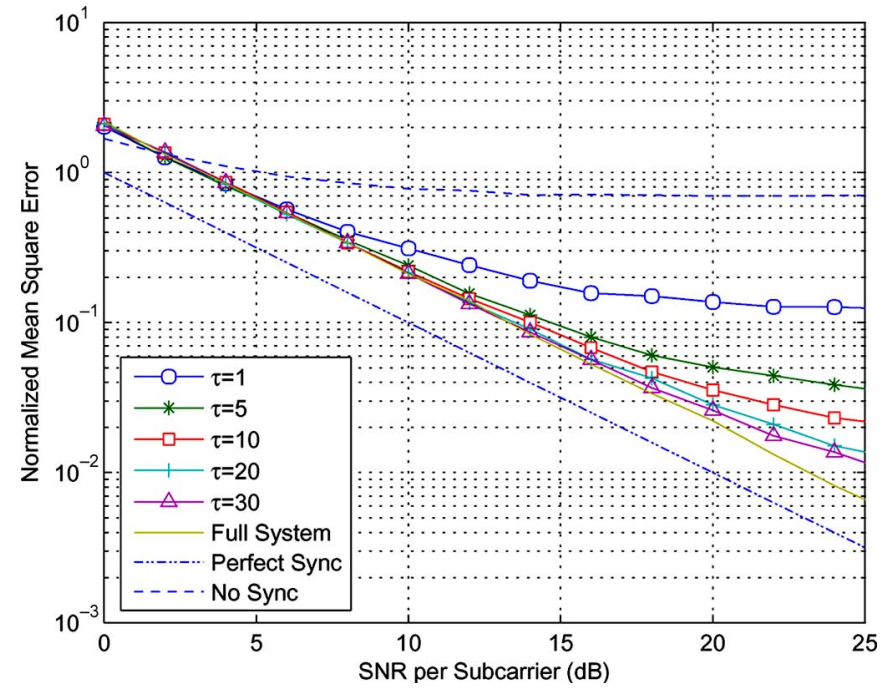

Fig. 6. nmse performance for the LS method based on full system and banded system with different $\tau$ 's. The DFT size is $N=512$, and there are $K=8$ users. The modulation scheme is QPSK.

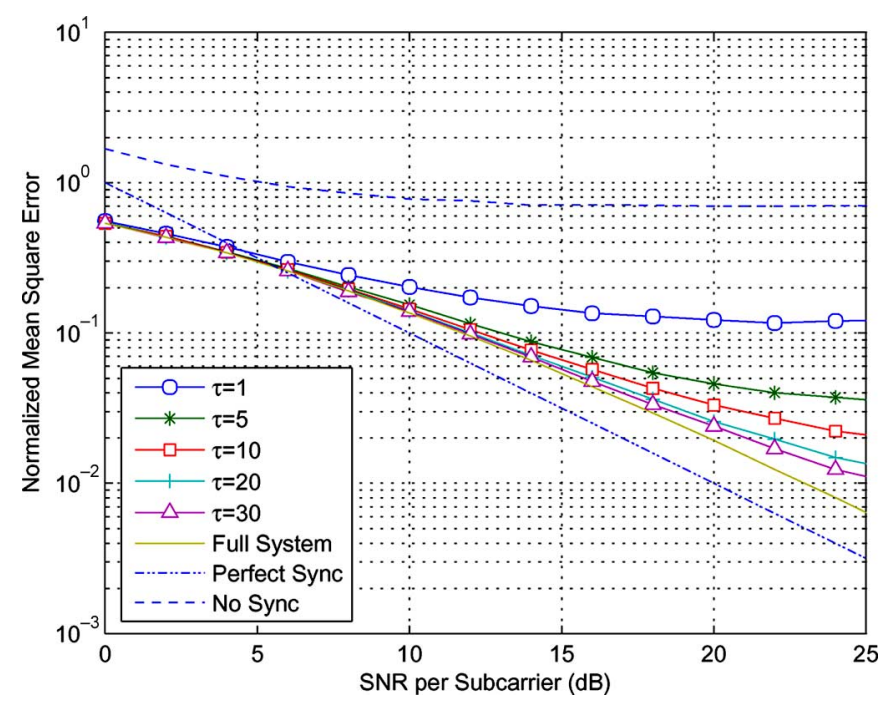

Fig. 7. nmse performance for the MMSE algorithm. The simulation parameters are the same as that in Fig. 6.

tation can provide a performance close to that of the full system for both algorithms when SNR $\leq 5 \mathrm{~dB}$. The performance floor of the banded system with $\tau=1$ manifests itself gradually with the increase of SNR. At 10-dB SNR, the performance of the banded system with $\tau=5$ is still comparable to that of the full system. The performances of the banded systems with $\tau=20$ and $\tau=30$ are very close to each other even at higher SNR. At uncoded BER of $10^{-2}$, the banded system $\tau=30$ has about 1-dB degradation compared with the full system implementation.

The simulation results discussed above also highlight the effect of the matrix bandwidth in different system scenarios. For wireless applications with typical operating SNR less than $15 \mathrm{~dB}$, a banded system with $\tau=5$ is suitable, since it maintains a performance comparable to that of the full system implementation and can save a significant amount of computational 


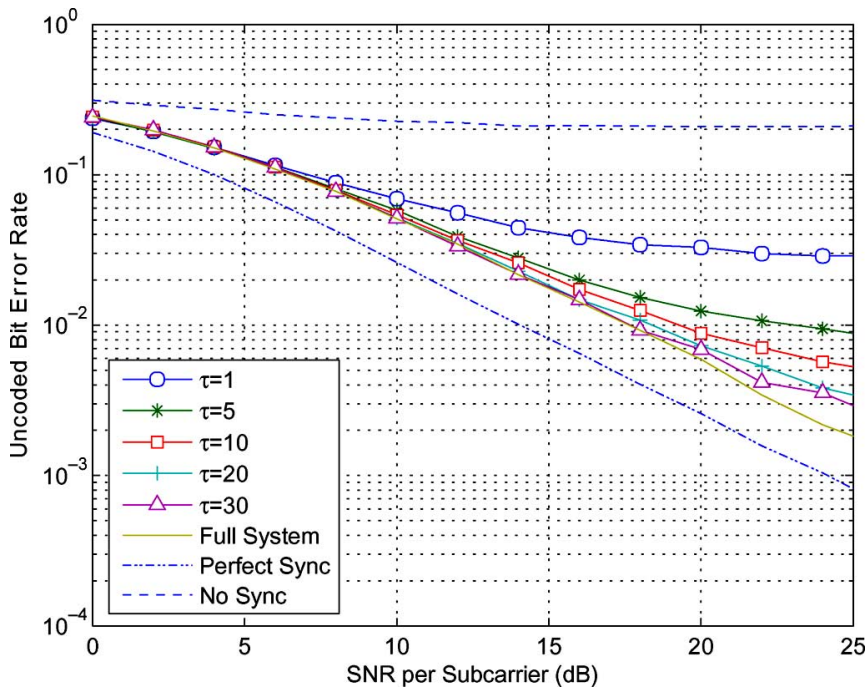

Fig. 8. Uncoded BER performance for the LS method. The simulation parameters are the same as that in Fig. 6.

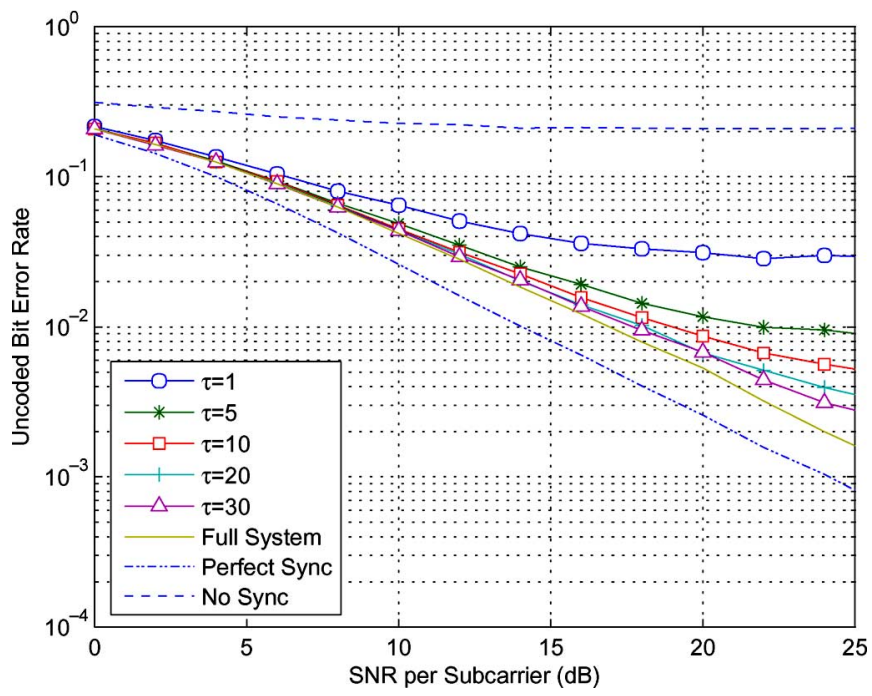

Fig. 9. Uncoded BER performance for the MMSE algorithm. The simulation parameters are the same as that in Fig. 6.

power. For systems operating in the SNR range of $15-20 \mathrm{~dB}$, banded systems with $\tau=20$ to 30 can provide acceptable tradeoff between computational complexity and performance.

High-order modulation constellations lead to high spectral efficiency. However, they also have stricter requirements on frequency synchronization accuracy. In Fig. 10, the uncoded BER performance with different constellations is studied for the same OFDMA system as that in Fig. 6 using $\tau=30$. In addition to QPSK and 16QAM, we also show the performance of a mixed setup in which four users are using QPSK and the other four are with 16QAM. For QPSK, MMSE outperforms LS by approximately $1 \mathrm{~dB}$ when the SNR is less than $10 \mathrm{~dB}$. The uncoded BER of both algorithms converge for SNR of more than $15 \mathrm{~dB}$. This is because the residual interference becomes a major factor in system performance. For 16QAM, the uncoded BERs of MMSE and LS are very close to each other for both high and low SNRs. As for the mixed setup, its performance is between QPSK and 16QAM.

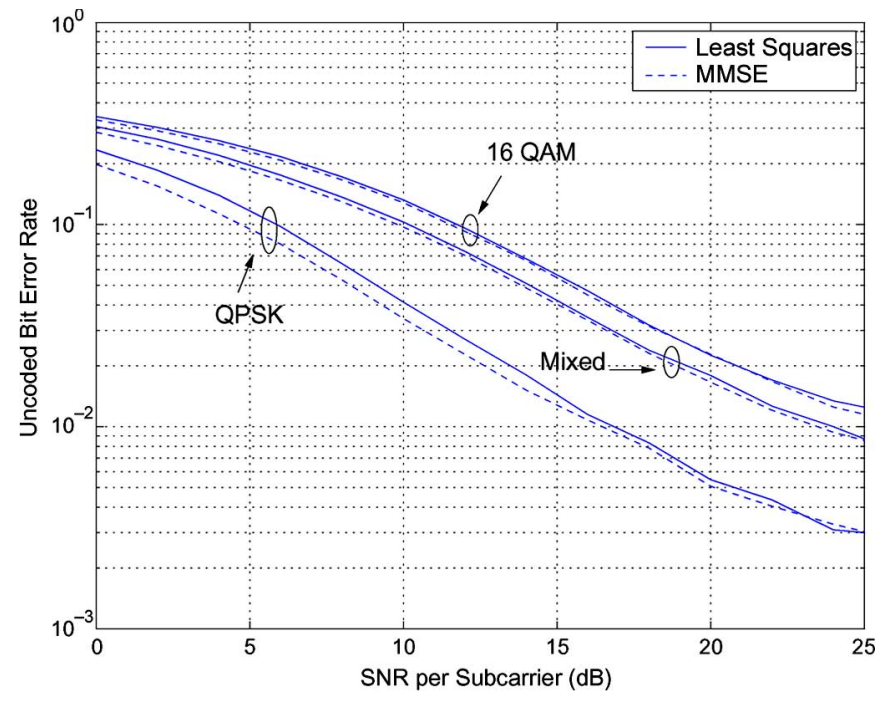

Fig. 10. Uncoded BER performance for different modulation constellations. $N=512,2 M=416$, and $K=8$. In the mixed setup, four users are with QPSK, and the other four are using 16QAM in an OFDMA block.

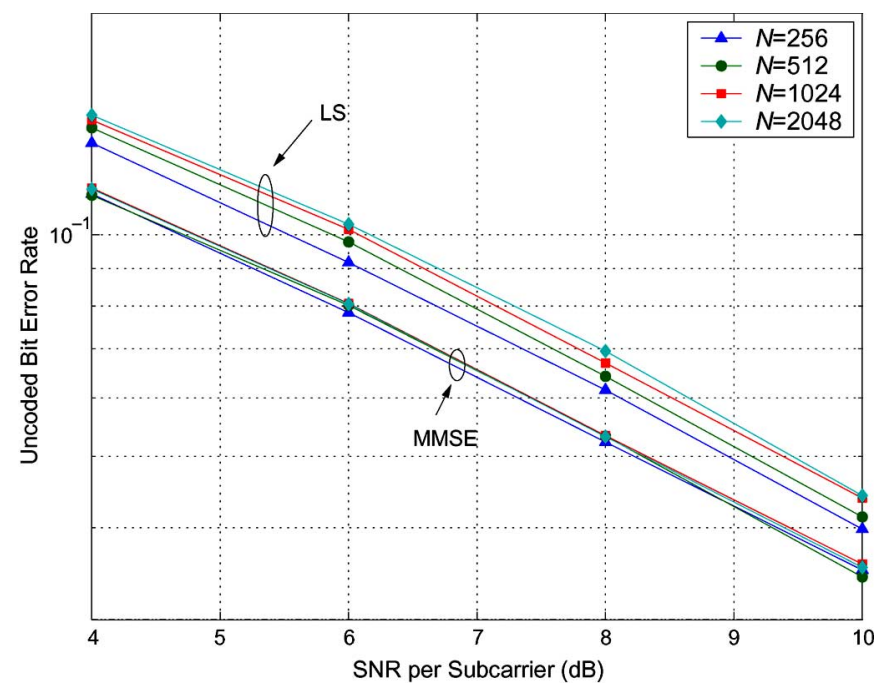

Fig. 11. Uncoded BER performance of the banded systems with the same bandwidth $\tau=30$ and different DFT sizes $N$.

The uncoded BER performance results of the banded systems with the same bandwidth $\tau=30$ but different DFT sizes are also studied, where $N$ is selected to be $256,512,1024$, and 2048. Corresponding to different DFT sizes, the available subcarriers are $212,424,848$, and 1696 , and the numbers of users in the system are $K=4,8,16,32$, respectively. The simulation results over the SNR range from 0 to $25 \mathrm{~dB}$ show that the performance results are close to each other, and the uncoded BER curves are not easily differentiable. In order to clarify the small difference, we plot the uncoded BER curves in a relatively small range in Fig. 11, where the SNR is from 4 to $10 \mathrm{~dB}$. The BER curves of MMSE for different $N$ 's are still clustered together and approximately $1.2 \mathrm{~dB}$ better than the LS for $N=2048$ and $0.7 \mathrm{~dB}$ better for $N=256$. For LS, the performance at $N=2048$ has about $0.5-\mathrm{dB}$ degradation compared to that for $N=256$. 


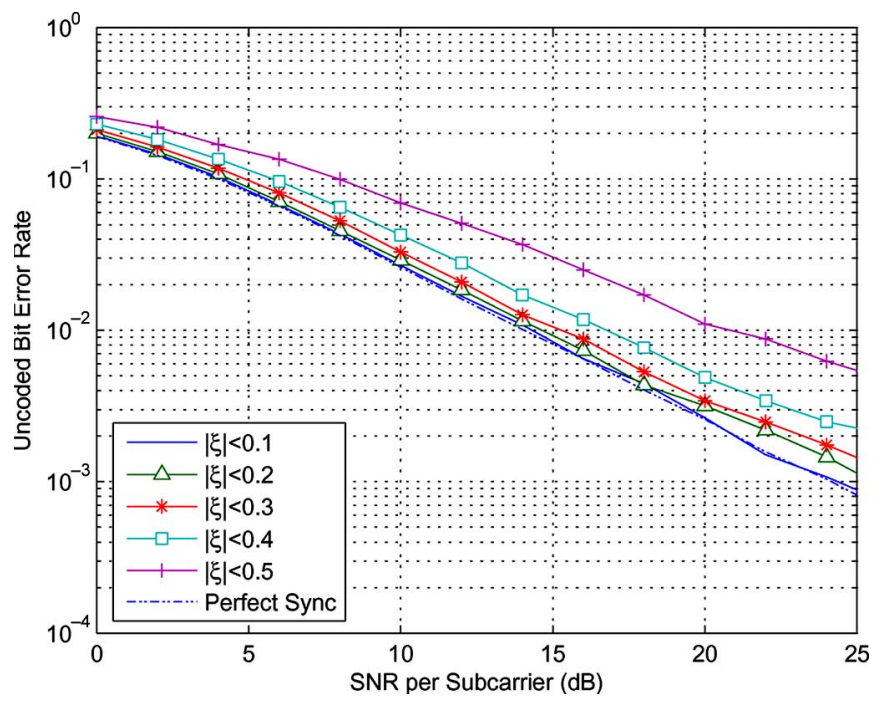

Fig. 12. Uncoded BER performance of the banded systems with $\tau=30$ and different NCFO range.

Finally, the performance of the banded system implementation under different NCFO ranges is studied in Fig. 12. For comparison, the uncoded BER with perfect synchronization is also presented. As can be expected, the smaller is the range of NCFO, the better is the performance. Note that when $|\xi|<0.1$, the performance of the banded implementation with $\tau=30$ almost overlapped with the perfect synchronized curve.

\section{CONCLUSION}

In this paper, the problem of frequency synchronization error is addressed for an OFDMA reverse link by constructing orthogonal spectral signals at the OFDMA UR. A generalized OFDMA uplink is described as the generalization of various subcarrier assignment schemes in OFDMA. A frequency domain signal model is formulated for the generalized OFDMA uplink signals based on an interference matrix, which characterizes the ICI due to multiuser frequency synchronization errors with respect to the common UR. LS and MMSE are applied, respectively, to construct the orthogonal spectral signals. Simulation results show that the proposed algorithms can realize appreciable performance improvement. Combining with the multiuser CFO estimator on OFDMA uplink [17], a solution for correcting the frequency synchronization errors on the OFDMA uplink is available for broadband wireless OFDMA systems to take advantages of its intrinsic frequency diversity and flexible resource allocation.

\section{APPENDIX}

\section{Proof of Full Rank Condition of $\boldsymbol{\Pi}$}

From (22), $\Pi$ can also be expressed as

$$
\boldsymbol{\Pi}=\mathbf{W}^{\mathcal{H}} \sum_{k=1}^{K} \mathbf{E}^{(k)} \mathbf{W} \Psi^{(k)} .
$$

Let $\mathbf{\Phi}=\sum_{k=1}^{K} \mathbf{E}^{(k)} \mathbf{W} \mathbf{\Psi}^{(k)}$. According to the definition of $\Psi^{(k)}$, if the $m$ th subcarrier belongs to user $k$, the $m$ th column of $\Phi$ is given by

$$
\phi_{m}= \begin{cases}\mathbf{E}^{(k)} \boldsymbol{w}_{m}, & \text { if } m \in \mathcal{M}_{q^{(k)}} \\ 0, & \text { otherwise }\end{cases}
$$

where $\boldsymbol{w}_{m}$ is the $i$ th column of $\mathbf{W}$, and $\mathbf{E}^{(k)}$ is given under (17). Therefore

$$
\phi_{m}=\left[1, e^{j 2 \pi\left(m+\xi^{(k)}\right) / N}, \ldots, e^{j 2 \pi\left(m+\xi^{(k)}\right)(N-1) / N}\right]^{\mathrm{T}}
$$

and $\boldsymbol{\Phi}$ is a Vandermonde matrix. The full rank condition of the Vandermonde matrix states that if $\boldsymbol{\Phi}$ is not a full rank, there exists two columns $m \in \mathcal{M}_{q^{(k)}}$ and $m^{\prime} \in \mathcal{M}_{q^{\left(k^{\prime}\right)}}$ such that

$$
m+\xi^{(k)}=m^{\prime}+\xi^{\left(k^{\prime}\right)} .
$$

Thus, $m-m^{\prime}=\xi^{\left(k^{\prime}\right)}-\xi^{(k)}$. Note that $m$ and $m^{\prime}$ are integers and mutually different, so $m-m^{\prime}$ is a nonzero integer. However, since $\left|\xi^{(k)}\right|<0.5$ and $\left|\xi^{\left(k^{\prime}\right)}\right|<0.5$, we have $\mid \xi^{\left(k^{\prime}\right)}-$ $\xi^{(k)} \mid<1$. Therefore, (A4) cannot hold, and $\boldsymbol{\Phi}$ is a full rank matrix. Note that $\mathbf{W}$ is also a full rank matrix; thus, $\Pi=$ $\mathbf{W}^{\mathcal{H}} \mathbf{\Phi}$ is a full rank matrix.

\section{ACKNOWLEDGMENT}

The authors would like to thank the reviewers and the editor of this paper for their valuable comments and suggestions.

\section{REFERENCES}

[1] Digital Audio Broadcasting (DAB) to Mobile, Portable and Fixed Receiver, May 2001. ETSI Std. ETS 300401.

[2] Digital Video Broadcasting (DVB-T); Frame Structure, Channel Coding, and Modulation for Digital Terrestrial Television, Dec. 2001. ETSI Std. ETS 300744

[3] Part 11: Wireless LAN Media Access Control (MAC) and Physical Layer (PHY) Specifications: High-Speed Physical Layer in the 5 GHZ Band, 2001. IEEE Std. 802.11a.

[4] Broadband Radio Access Network (BRAN): HIPERLAN Type 2 Functional Specification Part 1-Physical Layer, Jun. 1999. ETSI Std. ETS/BRAN 030 003-1.

[5] H. Rohling and R. Grunheid, "Performance of an OFDM-TDMA mobile communication system," in Proc. IEEE 46th Semiannual Veh. Technol. Conf., May 1996, pp. 1589-1593.

[6] J. Choi, C. Lee, H. W. Jung, and Y. H. Lee, "Carrier frequency offset compensation for uplink of OFDM-FDMA systems," IEEE Commun. Lett., vol. 4, no. 12, pp. 414-416, Dec. 2000.

[7] S. Hara and R. Prasad, "Overview of multicarrier CDMA," IEEE Commun. Mag., vol. 35, no. 12, pp. 126-133, Dec. 1997.

[8] D. Kivanc, G. Liu, and H. Liu, "Computationally efficient bandwidth allocation and power control for OFDMA," IEEE Trans. Wireless Commun., vol. 2, no. 6, pp. 1150-1158, Nov. 2003.

[9] Air Interface for Fixed Broadband Wireless Access Systems, 2004. IEEE Std. 802.16.

[10] I. Koffman and V. Roman, "Broadband wireless access solutions based on OFDM access in IEEE 802.16," IEEE Commun. Mag., vol. 40, no. 4, pp. 96-103, Apr. 2002.

[11] J. Bingham, "Multicarrier modulation for data transmission: An idea whose time has come," IEEE Commun. Mag., vol. 28, no. 5, pp. 5-14, May 1990.

[12] W. Rhee and J. M. Cioffi, "Increase in capacity of multiuser OFDM system using dynamic subchannel allocation," in Proc. IEEE Semiannual Veh. Technol. Conf., May 2000, pp. 1085-1089. 53th. 
[13] D. Galda, H. Rohling, and E. Costa, "On the effects of user mobility on the uplink of an OFDMA system," in Proc. IEEE Semiannual Veh. Technol. Conf.-Spring, 2003, vol. 2, pp. 1433-1437.

[14] A. M. Tonello, N. Laurenti, and S. Pupolin, "Analysis of the uplink of an asynchronous multi-user DMT OFDMA system impaired by time offsets, frequency offsets, and multi-path fading," in Proc. VTC Conf. Rec.-Fall, Oct. 2000, vol. 3, pp. 1094-1099.

[15] M. Morelli, "Timing and frequency synchronization for the uplink of an OFDMA system," IEEE Trans. Commun., vol. 52, no. 2, pp. 296-306, Feb. 2004.

[16] U. Tureli, H. Liu, and M. D. Zoltowski, "OFDM blind carrier offset estimation: ESPRIT,” IEEE Trans. Commun., vol. 48, no. 9, pp. 14591461, Sep. 2001

[17] Z. Cao, U. Tureli, and Y. Yao, "Deterministic multiuser carrier frequency offset estimation for interleaved OFDMA uplink," IEEE Trans. Commun., vol. 52, no. 9, pp. 1585-1594, Sep. 2004.

[18] J. J. van de Beek, P. O. Börjesson, M. L. Boucheret, D. Landström, J. Arenas, P. Ödling, C. Östberg, M. Mahlqvist, and S. K. Wilson, "A time and frequency synchronization scheme for multiuser OFDM," IEEE J. Sel. Areas Commun., vol. 17, no. 11, pp. 1900-1914, Nov. 1999.

[19] R. Nogueroles, M. Bossert, A. Donder, and V. Zyablov, "Improved performance of a random OFDMA mobile communication system," in Proc. 48th IEEE Semiannual Veh. Technol. Conf., May 1998, vol. 3, pp. 2502-2506.

[20] Y. Li and N. R. Sollenberger, "Clustered OFDM with channel estimation for high rate wireless data," IEEE Trans. Commun., vol. 49, no. 12, pp. 2071-2076, Dec. 2001.

[21] H. Niu, M. Shen, J. A. Ritcey, and H. Liu, "Performance of clustered OFDM with low density parity check codes over dispersive channels," in Proc. Rec. 36th Asilomar Conf. Signals, Syst., Comput., 2002, vol. 1, pp. $160-164$

[22] D. Huang and K. B. Letaief, "An interference-cancellation scheme for carrier frequency offsets correction in OFDMA systems communications," IEEE Trans. Commun., vol. 53, no. 7, pp. 1155-1165, Jul. 2005.

[23] P. Schniter, "Low-complexity equalization of OFDM in doubly selective channels," IEEE Trans. Signal Process., vol. 52, no. 4, pp. 1002-1011, Apr. 2004.

[24] A. Gorokhov and J.-P. Linnartz, "Robust OFDM receivers for dispersive time-varying channels: Equalization and channel acquisition," IEEE Trans. Commun., vol. 52, no. 4, pp. 572-583, Apr. 2004.

[25] M. S. El-Tanany, Y. Wu, and L. Hazy, "OFDM uplink for interactive broadband wireless: Analysis and simulation in the presence of carrier, clock and timing errors," IEEE Trans. Broadcast., vol. 47, no. 1, pp. 3-19, Mar. 2001

[26] X. Ma, C. Tepedelenliolu, G. B. Giannakis, and S. Barbarossa, "Nondata-aided carrier offset estimators for OFDM with null subcarriers: Identifiability, algorithms, and performance," IEEE J. Sel. Areas Commun., vol. 19 , no. 12 , pp. 2504-2515, Dec. 2001.

[27] D. Huang and K. B. Letaief, "Carrier frequency offset estimation for OFDM systems using null sub-carriers," IEEE Trans. Commun., vol. 54, no. 5, pp. 813-823, May 2006.

[28] M. Morelli, A. D’Andrea, and U. Mengali, "Frequency ambiguity resolution in OFDM systems," IEEE Commun. Lett., vol. 4, no. 4, pp. 134-136, Apr. 2000.

[29] P. H. Moose, "A technique for orthogonal frequency division multiplexing frequency offset correction," IEEE Trans. Commun., vol. 42, no. 10, pp. 2908-2914, Oct. 1994.

[30] S. Kapoor, D. J. Marchok, and Y.-F. Huang, "Adaptive interference suppression in multiuser wireless OFDM systems using antenna arrays," IEEE Trans. Signal Process., vol. 47, no. 12, pp. 3381-3391, Dec. 1999.

[31] J. Armstrong, "Analysis of new and existing methods of reducing intercarrier interference due to carrier frequency offset in OFDM," IEEE Trans. Commun., vol. 47, no. 2, pp. 365-369, Mar. 1999.

[32] Y. Zhao and S. G. Häggman, "Intercarrier interference self-cancellation scheme for OFDM mobile communication systems," IEEE Trans. Commun., vol. 49, no. 7, pp. 1185-1191, Jul. 2001.

[33] S. Haykin, Adaptive Filter Theory, 3rd ed. Englewood Cliffs, NJ: Prentice-Hall, 1996

[34] G. H. Golub and C. F. Van Loan, Matrix Computations, 3rd ed. Baltimore, MD: The Johns Hopkins Univ. Press, 1996.

[35] H. Huang, "Spatial channel model for multiple input multiple output (MIMO) simulations (Release 6)," 3GPP, Tech. Rep., 3GPP TR 25.996, Sep. 2003. V6.1.0. [Online]. Available: http://www.3gpp.org/ ftp/Specs/html-info/25996.htm

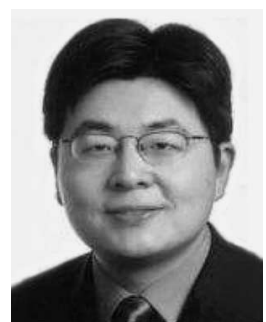

Zhongren Cao (S'01-M'04) received the B.Eng. degree from Xi' an Jiaotong University, Xi' an, China, in 1997, the M.Eng. degree from Shanghai Jiao Tong University, Shanghai, China, in 2000, and the Ph.D. degree from Stevens Institute of Technology, Hoboken, NJ, in 2004, all in electrical engineering.

$\mathrm{He}$ is currently an Assistant Project Scientist in the California Institute for Telecommunications and Information Technology, University of California, San Diego. He is leading a project team to prototype software-defined radio-based wireless networking testbed with emphasis on multicarrier and multiple antenna physical layer technology and cross-layer reconfigurability. His research interests are in the areas of communications, signal processing, networking, and software-defined radio.

Dr. Cao was a Reviewer for various IEEE Journals and Conferences, including IEEE TRANSACTIONS ON COMMUNICATIONS, IEEE TRANSACTIONS ON Wireless Communication, IEEE TRANSACtions ON Signal PROCESSING, International Conference on Communications (ICC), Globecom, Vehicular Technology Conference (VTC), Wireless and Networking Conference (WCNC), etc.

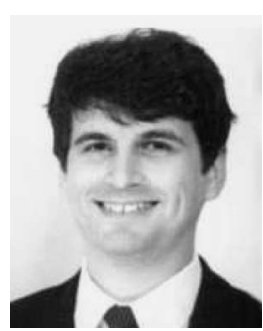

Ufuk Tureli (S'97-M'01) received the B.S. degree from Bogazici University, Istanbul, Turkey, in 1994, and the M.S. and Ph.D. degrees from the University of Virginia, Charlottesville, in 1998 and 2000, respectively, all in electrical engineering.

Since July 2000, he has been an Assistant Professor in the Department of Electrical and Computer Engineering, Stevens Institute of Technology, Hoboken, NJ. He is the Director of the Wireless Research Laboratory, Department of Electrical and Computer Engineering, and the Associate Director of the Wireless Network Security Center (WiNSeC), Stevens Institute of Technology. He has published numerous journal and conference articles in detection and estimation for scalable, adaptive, and robust broadband wireless communications. His research interests include signal processing with application to broadband wireless networks; estimation and detection for scalable, adaptive, and robust communications; and propagation studies.

Dr. Tureli serves on the Technical Program Committees of the IEEE Globecom, Milcom, ICC, and WCNC conferences, as well as the IEEE Communications Society (COMSOC) Radio Communications Committee. He is on the Editorial Board of the Elsevier Physical Communication (PHYCOM) Journal.

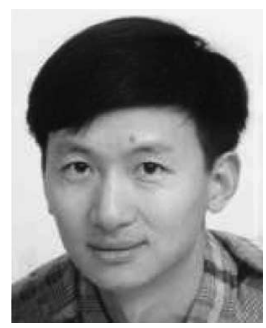

Yu-Dong Yao (S'88-M'88-SM'94) received the B.Eng. and M.Eng. degrees from Nanjing University of Posts and Telecommunications, Nanjing, China, in 1982 and 1985, respectively, and the Ph.D. degree from Southeast University, Nanjing, in 1988, all in electrical engineering.

Between 1989 and 1990, he was with Carleton University, Ottawa, ON, Canada, as a Research Associate working on mobile radio communications. Between 1990 and 1994, he was with Spar Aerospace Ltd., Montreal, QC, Canada, where he was involved in research on satellite communications. From 1994 to 2000, he was with Qualcomm Inc., San Diego, CA, where he participated in research and development in wireless CDMA systems. Since 2000, he has been with Stevens Institute of Technology, Hoboken, NJ, where he is currently an Associate Professor in the Department of Electrical and Computer Engineering and the Director of Wireless Information Systems Engineering Laboratory (WISELAB). He is the holder of one Chinese patent and seven U.S. patents. His research interests include wireless communications and networks, spread spectrum and CDMA, and DSP for wireless systems.

Dr. Yao is an Associate Editor of IEEE COMMUNICATIONS LETTERS and IEEE TRANSACTIONS ON VeHICULAR TECHNOLOGY, and an Editor of IEEE TRANSACTIONS ON Wireless COMMUNiCATIONS. He is a Guest Editor for a special issue on wireless networks for the International Journal of Communication Systems. 